\title{
Influence of dust on biogeochemical cycling in mountain ecosystems
}

\author{
SARAH M AARONS 1
}

1Scripps Institution of Oceanography, University of California San Diego, smaarons@ucsd.edu

Dust deposition is a critical control on soil nutrient supply and the distribution of terrestrial life. While the geologic record provides evidence of large fluctuations in dust flux and composition on glacial-interglacial timescales, rising temperatures and amplified droughts associated with climate change along with land use changes will undoubtedly impact the modern dust cycle. The degree that modern dust influences soil nutrient composition and supply is a topic of current contention, which may be partly attributable to the fact that the impact of dust is strongly dependent upon bedrock composition, local rates of soil production, and chemical erosion. Another complication is that dust fluxes and geochemical composition delivered to mountain ecosystems can vary with elevation and slope aspect. Here I will summarize current research shedding light on the role of dust on primary production and suggest what the research priorities should be in the future to improve model predictions of biogeochemical responses to increasing dust flux or changes in dust sources. 